\title{
Influência de Fatores Contingenciais na Adoção de Artefatos de Custos Tradicionais e Modernos na Rede Hoteleira do Paraná
}

\author{
Cleston Alexandre dos Santos ${ }^{a}$ \\ Vinícius Costa da Silva Zonatto ${ }^{b}$
}

\begin{abstract}
Resumo
A literatura sugere que os fatores contingenciais ambiente, tecnologia, estratégia, estrutura e porte organizacional, seja com maior ou menor intensidade, influenciam os atributos do sistema de contabilidade gerencial (SCG), mais especificamente na adoção de artefatos do sistema de custeio. No entanto, há poucas evidências entre tais relacionamentos especificamente no setor hoteleiro. Este artigo objetiva evidenciar a influência desses fatores na adoção de artefatos tradicionais e modernos de custos na rede hoteleira do estado do Paraná. Trata-se de um estudo descritivo, com procedimentos de interrogação, elaborado mediante a aplicação de pesquisa de campo realizada entre novembro e dezembro de 2015 com 40 hotéis. A análise dos dados utilizou estatística descritiva e correlação canônica. Os achados indicam que a maioria dos hotéis pesquisados adotam de forma plena os artefatos tradicionais de custos. Quanto aos modernos, boa parte dos investigados não tem planos para sua adoção, o que reforça a predominância de artefatos tradicionais nas organizações. Mesmo em hotéis de grande porte, em que se espera que os SCG apresentem estruturas mais sofisticadas, artefatos tradicionais como instrumento de suporte à tomada de decisão ainda têm sido utilizados. Conclui-se que os fatores contingenciais estrutura e estratégia influenciam mais na adoção de artefatos modernos de custos do que de tradicionais na rede hoteleira do estado do Paraná.
\end{abstract}

Palavras-chave: Fatores contingenciais; Artefatos de custos; Rede hoteleira; Paraná-Brasil.

\section{Abstract \\ The influence of contingency factors on the adoption of traditional and modern cost artifacts in the hotel network of the state of Paraná}

The literature suggests that the contingency factors: environment, technology, strategy, structure and organizational size, with greater or lesser intensity, influence the attributes of the management accounting system, more specifically in the adoption of costing system artifacts. Nonetheless, there is little evidence about such relationships in the hotel sector. The present article has as main objective to evidence the influence of these factors on the adoption of traditional and modern cost artifacts in the hotel industry of the state of Paraná, Brazil. It is a descriptive study with survey approach, carried out between the months of November and December 2015 with 40 hotels. Data were analyzed statistically using descriptive statistics and canonical correlation. Findings indicate that the majority

a. Doutor em Ciências Contábeis e Administração pela Universidade Regional de Blumenau, Blumenau, Santa Catarina, Brasil. Professor do departamento de Ciências Contábeis da Universidade Federal de Mato Grosso do Sul, Três Lagoas, Mato Grosso do Sul, Brasil. E-mail: cleston.alexandre@hotmail.com

b. Possui pós-doutorado em Ciências Contábeis pela Universidade do Vale do Rio dos Sinos, São Leopoldo, Rio Grande doSul, Brasil.Professor do departamento de Ciências Contábeis da Universidade Federal de Santa Maria, Santa Maria, Rio Grande do Sul, Brasil. E-mail: viniciuszonatto@gmail.com 
of the hotels fully implement traditional cost artifacts. Regarding modern cost artifacts, considerable part of the investigated hotels has no plans for adopting and implementing them, a fact that reinforces the predominance of adoption of traditional artifacts in these organizations. It was expected that management accounting systems presented more sophisticated structures, but even in large hotels, data revealed that traditional artifacts have still been used as supportive instruments for the decision-making process. Findings lead to the conclusion that the contingency factors structure and strategy influence more in the adoption of modern cost artifacts than traditional artifacts in the hotel industry of the state of Paraná, Brazil.

Keywords: Contingency factors; Cost artifacts; Hotel industry; Paraná, Brazil.

\section{Resumen}

Influencia de los factores de contingencia en el adopción de artefactos de costos tradicional y moderno en la rede hotelera de Paraná

La literatura sugiere que los factores contingenciales: ambiente, tecnología, estrategia, estructura y tamaño de la organización, con mayor o menor intensidad, influencien en los atributos del sistema de contabilidad de gestión, más específicamente en el adopción de artefactos del sistema de costeo. Sin embargo, existen pocas evidencias de tales relaciones, específicamente en el sector hotelero. Este artículo tiene como objetivo evidenciar la influencia de estos factores en el adopción de artefactos de costos tradicionales y modernos del sector hotelero de Paraná, Brasil. Se trata de un estudio de campo descriptivo con adopción de cuestionario, realizado entre noviembre y diciembre de 2015 en 40 hoteles. Los datos fueron analizados mediante estadística descriptiva y Correlación Canónica. Los resultados indican que la mayoría de los hoteles investigados utiliza plenamente los artefactos tradicionales de costos. En cuanto a los artefactos modernos de costos, la mayor parte de los investigados no tienen planes para su adopción, lo que refuerza el predominio del adopción de artefactos tradicionales en detrimento de los modernos en esas organizaciones. Incluso, en los grandes hoteles, donde se espera que el SGC presente estructuras más sofisticadas, la presencia de artefactos tradicionales como instrumentos de soporte a la toma de decisiones aún es utilizada. Se concluye que los factores contingenciales estructura y estrategia influencian más en el adopción de artefactos modernos de costos que de artefactos tradicionales en redes hoteleras de Paraná, Brasil.

Palabras clave: Factores de contingencia; Artefactos de costos; Redes hoteleras; Paraná, Brasil.

\section{INTRODUÇÃO}

Os avanços tecnológicos, a globalização, as mudanças no contexto social, como também o mercado competitivo, provocam incertezas no processo decisório das organizações, o que pode influenciar a sua permanência no mercado junto aos concorrentes, inclusive as entidades que compreendem as redes hoteleiras (Beuren \& Fiorentin, 2014). 0 cenário organizacional é composto e influenciado por vários fatores, os quais precisam ser controlados e ajustados pelos gestores. Nesse contexto, Otley (1980) destaca que os gestores, ao estarem cientes dos fatores que influenciam as organizações e considerando que estes possam ser controláveis, buscam adotar artefatos de controle gerencial e configurações organizacionais que possibilitem melhores resultados. 
Tais relacionamentos são investigados na literatura contábil a partir da abordagem teórica da teoria da contingência. Por meio dela e do ambiente em que as organizações estão inseridas, procura-se compreender e gerar uma explicação de como determinados fatores impactam no seu crescimento e permanência no mercado (Beuren \& Fiorentin, 2014), bem como se tais circunstâncias afetam a estrutura do sistema de controle das empresas (Chenhall, 2007). Beuren e Fiorentin (2014) destacam que, pelo fato de as organizações funcionarem em diferentes condições, que variam em função do ambiente em que pertencem, essas condições, ou fatores contingenciais, podem ser caracterizadas como ameaças ou oportunidades que afetam diretamente a estrutura e os processos internos das entidades.

Evidências encontradas na literatura apontam que um ambiente cada vez mais competitivo, que tem influenciado mudanças na estrutura organizacional, em tecnologia de serviços e nas práticas de custeio, exige maior foco nas estratégias de diferenciação (Baines \& Langfield-Smith, 2003). Também sugere que a teoria da contingência pode contribuir para compreender qual sistema de contabilidade gerencial (SCG) é adotado dentro do seu contexto organizacional (Chenhall, 2007). Da mesma forma, auxilia no entendimento de como os fatores contingenciais ambiente, tecnologia, estratégia, estrutura e porte organizacional, seja com maior ou menor intensidade, influenciam os atributos do SCG (Beuren \& Fiorentin, 2014), bem como a decisão de uso do sistema de custeio, que compreende artefatos tradicionais e modernos de contabilidade gerencial (Silva et al., 2014).

Por artefatos de contabilidade gerencial, sejam tradicionais ou modernos, entende-se como sendo sistemas de informação, modelos de gestão, sistemas de custeio, filosofias implantadas pelas organizações e conceitos de mensuração e avaliação de desempenho, que possam ser explorados pelos gestores no desenvolvimento de suas atividades (Frezatti, 2005; Guerreiro, Cornachione Júnior, \& Soutes, 2011). Os artefatos tradicionais e modernos de contabilidade gerencial têm estimulado o desenvolvimento de novas pesquisas na área contábil. Em especial, buscando avaliar se os instrumentos de controle gerencial utilizados pelas organizações têm evoluído e se são adequados às necessidades dos gestores. Neste contexto, considerando os diferentes artefatos identificados na literatura (Frezatti, 2005; Guerreiro et al., 2011; Soutes, 2006), para a realização deste estudo aborda-se mais especificamente aqueles relacionados aos sistemas de custeio.

Foram considerados como artefatos tradicionais para a gestão de custos o Custeio Padrão, o Custeio por Absorção e o Custeio Variável. Já como artefatos modernos, foram considerados o Custeio Baseado em Atividades (ABC), Custeio Baseado em Atividade e Tempo (TDABC) e o Custeio Meta (Frezatti, 2005; Guerreiro et al., 2011; Leitão \& Silva, 2009; Lima, Egito, \& Silva, 2004; Maher, 2001; Soutes, 2006; Soutes \& Zen, 2005; Souza, Lisboa, \& Rocha, 2003).

A literatura tem mostrado, especificamente no setor hoteleiro do país, que há poucos resultados que permitam concluir sobre relacionamentos entre fatores contingenciais e artefatos tradicionais e modernos de custos. Este setor tem sido apontado como uma das atividades destacadas pelo seu crescimento econômico nos últimos anos (Marquesan, Guzzo, Zawislak, \& Tello-Gamarra, 2015), constituindo-se como uma importante área de pesquisa no Brasil. Este segmento é caracterizado pelo elevado grau de competição entre as organizações hoteleiras concorrentes, o que exige dos gestores o estabelecimento de ações que oportunizem uma melhor tomada de decisão, com o propósito de promover o desenvolvimento 
de suas empresas (Tsai, Song, \& Wong, 2009; Wilke \& Rodrigues, 2013; Zonatto, Schuh, \& Zonatto, 2014). Tais condições são adequadas para esta investigação.

Nesse contexto, levando em consideração as variáveis contingenciais citadas e tendo em vista a importância dos sistemas de custeio no processo gerencial das organizações hoteleiras, para a realização deste estudo estabeleceu-se a seguinte questão de pesquisa: Qual a influência de fatores contingenciais na adoção de artefatos tradicionais e modernos de custos na rede hoteleira do estado do Paraná? Assim, o objetivo desta pesquisa consiste em verificar a influência de fatores contingenciais na adoção de artefatos tradicionais e modernos de custos na rede hoteleira do estado do Paraná.

Em situações que envolvem contingências a organização pode ter uma instabilidade, gerando desequilíbrio na sua gestão, o que pode acarretar alterações nos sistemas de custeio, com o propósito de ajustar a situação da organização ao ambiente inserido (Silva et al., 2014). Guerreiro, Frezatti, Lopes e Pereira (2005) apontam que pesquisas em torno das mudanças do SCG relacionadas à teoria da contingência têm sido pouco estudadas na área contábil, fato este que abre novas oportunidades de investigações, inclusive no estado do Paraná.

De acordo com Píccolo e Gândara (2012), a expansão das redes hoteleiras no estado do Paraná tem sido contínua. De maneira geral, tem ocorrido devido ao crescimento econômico das cidades e ao desenvolvimento do turismo de negócios, como também pelo turismo de lazer em várias cidades, pois esses fatores (crescimento econômico e de desenvolvimento do turismo) acabam criando uma demanda permanente de homens de negócios para esses destinos. Dessa forma, o fato de identificar e entender a influência de fatores contingenciais nos artefatos de custos na rede hoteleira do estado do Paraná possibilita melhorias e avanços na gestão dessas organizações.

\section{FUNDAMENTAÇÃo TEÓRICA}

\section{Gestão hoteleira e artefatos de custos}

Os hotéis fazem parte do sistema turístico, de modo que suas atividades são diretamente relacionadas ao turismo e essenciais para sua continuidade. As redes hoteleiras pertencem a um setor extremamente competitivo, sendo que há uma vantagem das redes com relação aos estabelecimentos locais quando se trata de tecnologia avançada (Píccolo \& Gândara, 2012).

Nas duas últimas décadas, as organizações, inclusive as de atividade hoteleira, têm sofrido mudanças significativas na estrutura organizacional, envolvendo principalmente os aspectos de competitividade e tecnologias de informação (Burns \& Vaivio, 2001). A atividade hoteleira exige atenção dos gestores na condução dos negócios, principalmente em função da questão estratégica, por exemplo, na discussão da definição de preços dos serviços versus sazonalidade. Diante do exposto, torna-se necessário identificar as particularidades do setor para prover artefatos gerenciais adequados e em tempo hábil, e assim conduzir para uma boa gestão da atividade, já que a ausência de informações gerenciais ou transmitidas de forma inadequada pode comprometer o processo decisório do gestor e, por consequência, o sucesso da entidade (Sharma, 2002). 
Para possibilitar aos gestores de hotéis informação adequada e relevante para competirem num ambiente de plena mudança, a contabilidade gerencial, incluindo os sistemas de custos, precisa mudar, no sentido de analisar a real necessidade e o melhor artefato contábil gerencial para cada organização (Faria, Trigueiros, \& Ferreira, 2012). Sharma (2002) já chamava a atenção para a necessidade de os gestores conhecerem melhor a estrutura organizacional dos hotéis a fim de indicarem o melhor sistema de controle de gestão, dada a relevância econômica da indústria hoteleira em muitas economias mundiais.

Espejo, Costa, Cruz e Almeida (2009) argumentam que, para alcançar os objetivos organizacionais pré-estabelecidos, é necessário que o SCG, neste caso, por meio de seus artefatos tradicionais e modernos de custos, leve em consideração todas as diferenças das necessidades informacionais dos usuários. Neste estudo, como artefatos tradicionais de custos foram considerados o Custeio Padrão, o Custeio por Absorção e o Custeio Variável e como artefatos modernos, o ABC, o TDABC e o Custeio Meta (Frezatti, 2005; Guerreiro et al., 2011; Leitão \& Silva, 2009; Lima et al., 2004; Maher, 2001; Soutes, 2006; Soutes \& Zen, 2005; Souza et al., 2003). A Quadro 1 apresenta as características de cada um destes artefatos de custos.

\section{Quadro 1 - Características dos artefatos tradicionais e modernos de custos}

\begin{tabular}{|c|c|c|}
\hline \multicolumn{2}{|c|}{ Artefato } & Características \\
\hline \multirow{3}{*}{ 矛 } & $\begin{array}{l}\text { Custeio } \\
\text { por } \\
\text { absorção }\end{array}$ & $\begin{array}{l}\text { Consiste na alocação de todos os custos de produção ou custos de prestação } \\
\text { de serviço aos bens ou serviços produzidos, sendo os custos diretos por meio } \\
\text { da apropriação direta e os indiretos por meio de critérios de rateios. }\end{array}$ \\
\hline & $\begin{array}{l}\text { Custeio } \\
\text { padrão }\end{array}$ & $\begin{array}{l}\text { Trata-se de um método em que o custo de produção/prestação de serviço é } \\
\text { estabelecido antes do início do processo produtivo, sendo determinado com } \\
\text { base em informações de períodos anteriores e experiências simuladas que } \\
\text { levam em consideração as deficiências existentes nos fatores de produção/ } \\
\text { prestação de serviço, disponíveis dentro do processo produtivo de cada } \\
\text { organização. }\end{array}$ \\
\hline & $\begin{array}{c}\text { Custeio } \\
\text { variável }\end{array}$ & $\begin{array}{l}\text { Consiste na identificação dos custos variáveis e fixos, ou seja, aqueles que } \\
\text { oscilam de forma proporcional ao volume de produção e vendas/prestação } \\
\text { de serviços e os custos que se mantém estáveis perante o volume de } \\
\text { produção e vendas/prestação de serviços. Nesse método são alocados aos } \\
\text { bens e serviços produzidos apenas os custos variáveis de produção (diretos } \\
\text { e indiretos). }\end{array}$ \\
\hline \multirow{3}{*}{ 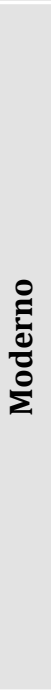 } & $\mathrm{ABC}$ & $\begin{array}{l}\text { Consiste na identificação, análise e controle dos custos envolvidos nas } \\
\text { atividades e processos de uma organização. Nesse método, identifica-se as } \\
\text { atividades e os processos existentes nos setores produtivos/prestação de } \\
\text { serviços de uma organização industrial ou prestadora de serviço, alocando } \\
\text { os custos aos produtos por meio da utilização dos direcionadores ou } \\
\text { geradores de custos dessas atividades. }\end{array}$ \\
\hline & TDABC & $\begin{array}{l}\text { Esse método necessita da estimativa de apenas duas variáveis para sua } \\
\text { operacionalização: o custo de fornecimento de recursos a determinada } \\
\text { atividade e o tempo demandado para execução. Tem como objetivo principal } \\
\text { reduzir a dificuldade na apuração de vários direcionadores de recursos, ao } \\
\text { reduzi-los para apenas um, que é o tempo. }\end{array}$ \\
\hline & $\begin{array}{c}\text { Custeio } \\
\text { meta }\end{array}$ & $\begin{array}{l}\text { Nesse método, o custeio é realizado por meio da subtração de um preço } \\
\text { estimado (ou preço de mercado) da margem de lucratividade almejada, } \\
\text { tendo como propósito alcançar um custo de produção desejado. Resumindo, } \\
\text { o custo meta é calculado levando em consideração o preço de venda menos o } \\
\text { lucro desejado. }\end{array}$ \\
\hline
\end{tabular}

Guerreiro et al. (2011) 


\section{Fatores contingenciais}

Ao considerar os pressupostos da abordagem sistêmica, a teoria da contingência demonstra que a organização é caracterizada como um sistema aberto, de tal forma que a variável externa ambiente, de maneira independente, exerce influência nas variáveis internas, como tecnologia, estrutura, porte organizacional e estratégia (Espejo, 2008). É observado que os fatores contingenciais, como o ambiente, os aspectos comportamentais, que compreendem a resistência às mudanças e os estilos de liderança, assim como tecnologia, estrutura, porte organizacional e estratégia, podem ser determinantes para as atividades econômicas (Baines \& Langfield-Smith, 2003; Espejo, 2008).

0 ambiente é considerado o fator contingencial externo à organização, que segundo Chenhall (2003, p. 136), pode ser caracterizado como sendo "atributos particulares como intensa competição de preço de competidores potenciais ou existentes, ou a probabilidade de uma mudança na disponibilidade ou diferenciação de serviços". Já quanto à contingência tecnologia, compreende o modo como são realizados os processos da organização, o que inclui o conhecimento das atividades do negócio, as pessoas envolvidas, hardware e software (Chenhall, 2003). Hyvönen (2007, p. 353) define-a como sendo "o uso de inovações de tecnologia da informação em geral".

O fator contingencial estrutura, tratado como elemento interno e controlável, é considerado a maneira com que as organizações evidenciam sua autoridade e as responsabilidades dos colaboradores. Essa variável mostra como a divisão das atividades e as atribuições de autoridades são divididas visando os objetivos organizacionais (Maximiano, 2004; Sharma, 2002). Espejo (2008) argumenta que a existência de maior grau de descentralização na estrutura da empresa representa que ela tende a ser mais orgânica. Em contrapartida, quando sua estrutura contempla a centralização de atividades, ela tende a ser configurada como mais mecanicista.

0 porte organizacional é um dos fatores que impacta na estrutura e nas formas de controles das organizações. Maiores empresas detêm mais recursos do que as menores para implantação de práticas de gestão mais modernas (Abdel-Kader \& Luther, 2008). Chenhall (2003) argumenta que a maioria dos estudos desenvolvidos na área contábil investigam apenas as grandes organizações, justificando que elas incorporam práticas vinculadas a um sistema de controle gerencial considerado como mais formal.

Por fim, o fator estratégia é definido como sendo "um conjunto de regras de tomada de decisão para orientação do comportamento de uma organização" (Ansoff \& Mcdonnell, 1993, p. 70). Chenhall (2007) caracteriza a estratégia como sendo o fator pelo qual os gestores tornam-se influenciados pela natureza do ambiente externo, como também pela cultura, controle para tomar decisão e os mecanismos estruturais.

Evidências encontradas na literatura sugerem que a contabilidade tem recuperado sua relevância e respondido às necessidades informacionais das organizações, por meio de novos artefatos contábeis e alterações nos sistemas de gestão utilizados pelas empresas, os quais são capazes de produzir informações que oportunizam uma melhor tomada de decisão ao gestor, 
adequando a instabilidade organizacional às demandas de mercado. Por consequência, possibilitam ao gestor uma atuação mais coerente ao processo decisório (Johnson \& Kaplan, 1993). Contudo, também há resultados de estudos desenvolvidos sobre essa temática que sugerem que, apesar dos benefícios que podem ser alcançados a partir do uso de tais técnicas modernas de gestão, sua efetiva adoção não tem sido generalizada (Chenhall \& LangfieldSmith, 1998; Faria et al., 2012). Tal gap tem estimulado a realização de novos estudos na área contábil.

Neste contexto, considerando-se tais apontamentos, espera-se que os fatores contingenciais ambiente, tecnologia, estratégia, estrutura e porte organizacional, seja com maior ou menor intensidade, influenciem de alguma forma o SCG adotado por empresas hoteleiras no estado do Paraná, mais especificamente no que se refere à decisão de uso do sistema de custeio (Beuren \& Fiorentin, 2014; Silva et al., 2014). Como o crescimento econômico do setor hoteleiro tem sido de forma contínua, ele tem vivenciado mudanças significativas na estrutura organizacional (Burns \& Vaivio, 2001; Marquesan et al., 2015).

Além do evidenciado, espera-se também que questões ambientais como concorrência, tecnologia, gostos e preferências do cliente; questões tecnológicas como comércio eletrônico e gerenciamento da relação com o cliente; questões de estrutura como ampliação ou oferta de novos serviços e investimentos relevantes; organizações de porte elevado; e fatores de estratégia como alta qualidade do serviço e características diferenciadas do serviço com imagem da organização reconhecida no mercado, influenciem na adoção de artefatos de custos diferenciados, que sejam considerados atuais e relevantes (Baines \& Langfield-Smith, 2003; Beuren \& Fiorentin, 2014; Chenhall, 2007; Cooper \& Kaplan, 1998; Faria et al., 2012; Guerra, 2007; Rodniski \& Souza, 2014; Silva et al., 2014).

Diante desses argumentos, para a realização desta pesquisa, estabeleceu-se a hipótese geral de que os fatores contingenciais influenciam mais na adoção de artefatos modernos de custos do que artefatos tradicionais na rede hoteleira do estado do Paraná. Para uma análise mais específica dos fatores contingenciais, estabeleceram-se as seguintes hipóteses:

H1: O fator contingencial ambiente influencia mais na adoção de artefatos modernos de custos do que artefatos tradicionais na rede hoteleira do estado do Paraná.

H2: O fator contingencial tecnologia influencia mais na adoção de artefatos modernos de custos do que artefatos tradicionais na rede hoteleira do estado do Paraná.

H3: O fator contingencial estrutura influencia mais na adoção de artefatos modernos de custos do que artefatos tradicionais na rede hoteleira do estado do Paraná.

H4: O fator contingencial porte organizacional influencia mais na adoção de artefatos modernos de custos do que artefatos tradicionais na rede hoteleira do estado do Paraná.

H5: O fator contingencial estratégia influencia mais na adoção de artefatos modernos de custos do que artefatos tradicionais na rede hoteleira do estado do Paraná. 


\section{METODOLOGIA}

Neste tópico são apresentados os procedimentos metodológicos da pesquisa. Com base em Cooper e Schindler (2003), pode-se afirmar que a pesquisa aborda procedimentos de interrogação por meio de questionário, além de tratar-se de um estudo formal, ex post facto, descritivo, transversal, estatístico e também em ambiente de campo.

Para este trabalho, têm-se como população 104 hotéis do estado do Paraná, vinculados à Associação Brasileira da Indústria de Hotéis do Estado do Paraná (ABIH-PR). A amostra foi composta por 40 hotéis que voluntariamente responderam à pesquisa, tratando-se assim de uma amostra não aleatória, que abrangeu 38,46\% da população objeto de estudo. A amostra classifica-se como não probabilística, alcançada por acessibilidade, pelo fato de os questionários terem sido enviados a todos os hotéis que compõem a população efetiva e de não ter como definir de forma prévia quais participarão (Fonseca \& Martins, 1996). A Quadro 2 apresenta o constructo da pesquisa.

Quadro 2 - Constructo da pesquisa

\begin{tabular}{|c|c|c|c|c|}
\hline Categoria & Subcategoria & Questão & Proxy & Autores \\
\hline \multirow{2}{*}{ 를 } & $\begin{array}{l}\text { Caracterização } \\
\text { do respondente }\end{array}$ & 01 a 07 & Alternativas & \multirow[b]{2}{*}{$\begin{array}{c}\text { Elaborado pelos } \\
\text { autores. }\end{array}$} \\
\hline & $\begin{array}{c}\text { Caracterização } \\
\text { do hotel }\end{array}$ & 08 a 12 & Alternativas & \\
\hline \multirow{5}{*}{ 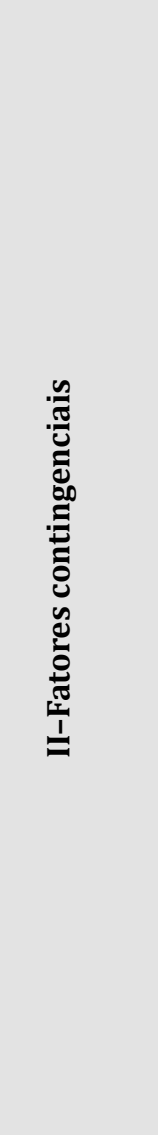 } & $\begin{array}{l}\text { Variável } \\
\text { ambiente }\end{array}$ & 13 a 17 & $\begin{array}{l}\text { Escala de } 1 \text { a 5: muito estável } \\
\text { (1); estável (2); intermediário } \\
\text { (3); dinâmico (4); muito } \\
\text { dinâmico (5). }\end{array}$ & \multirow{3}{*}{$\begin{array}{l}\text { Gordon e } \\
\text { Narayanan } \\
\text { (1984), Chenhall } \\
\text { e Langfield-Smith } \\
\text { (1998), Sharma } \\
\text { (2002), Hansen } \\
\text { e Van der Stede } \\
\text { (2004), Hyvönen } \\
\text { (2007), Dekker, } \\
\text { Groot e Schoute } \\
\text { (2012) e Espejo } \\
\text { (2008). }\end{array}$} \\
\hline & $\begin{array}{l}\text { Variável } \\
\text { tecnologia }\end{array}$ & 18 a 21 & $\begin{array}{l}\text { Escala de } 1 \text { a } 5 \text { : não há uso (1); } \\
\text { está estudando a viabilidade } \\
\text { de uso (2); uso em baixa } \\
\text { intensidade (3); uso em } \\
\text { moderada intensidade (4); uso } \\
\text { em grande intensidade (5). }\end{array}$ & \\
\hline & $\begin{array}{l}\text { Variável } \\
\text { estrutura }\end{array}$ & 22 a 26 & $\begin{array}{l}\text { Escala de } 1 \text { a 5: nunca delega } \\
\text { (1); já delegou, mas não } \\
\text { delega mais (2); delega pouco } \\
\text { (3); delega frequentemente } \\
\text { (4); delegação total (5). }\end{array}$ & \\
\hline & Variável porte & 27 & $\begin{array}{l}\text { Escala de } 1 \text { a } 3 \text { : até } 50 \\
\text { unidades habitacionais (UH) } \\
\text { (1); de } 51 \text { a } 100 \text { UH (2); acima } \\
\text { de } 100 \text { UH (3). }\end{array}$ & $\begin{array}{l}\text { Silva }(2000) \text { e } \\
\text { Espejo (2008). }\end{array}$ \\
\hline & $\begin{array}{l}\text { Variável } \\
\text { estratégia }\end{array}$ & 28 a 31 & $\begin{array}{l}\text { Escala de } 1 \text { a } 5 \text { : não } \\
\text { importante (1); pouco } \\
\text { importante (2); importante } \\
\text { (3); muito importante (4); } \\
\text { extremamente importante (5). }\end{array}$ & $\begin{array}{l}\text { Chenhall e } \\
\text { Langfield-Smith } \\
\text { (1998), Hansen } \\
\text { e Van der Stede } \\
\text { (2004) e Espejo } \\
\text { (2008). }\end{array}$ \\
\hline
\end{tabular}

(continua...) 
Quadro 2 - Continuação

\begin{tabular}{|c|c|c|c|c|}
\hline Categoria & Subcategoria & Questão & Proxy & Autores \\
\hline \multirow{2}{*}{ 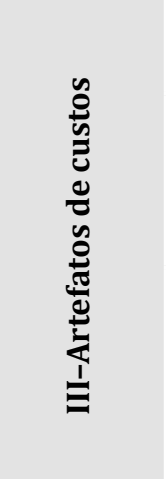 } & $\begin{array}{l}\text { Artefatos } \\
\text { tradicionais }\end{array}$ & 32 a 34 & $\begin{array}{l}\text { Escala de } 5 \text { pontos: não } \\
\text { há planos para adotar (1); } \\
\text { avaliou e descartou a adoção } \\
\text { (2); em processo de avaliação } \\
\text { (3); adoção inicial (4); adoção } \\
\text { plena (5). }\end{array}$ & $\begin{array}{l}\text { Maher (2001), } \\
\text { Souza, Lisboa e } \\
\text { Rocha (2003), } \\
\text { Soutes (2006). }\end{array}$ \\
\hline & $\begin{array}{c}\text { Uso e finalidade } \\
\text { dos artefatos }\end{array}$ & 39 a 44 & $\begin{array}{l}\text { Escala de } 5 \text { pontos: discordo } \\
\text { totalmente (1); discordo (2); } \\
\text { nem discordo e nem concordo } \\
\text { (3); concordo (4); concordo } \\
\text { totalmente (5); e alternativas. }\end{array}$ & $\begin{array}{l}\text { Abdel-Kader e } \\
\text { Luther (2006) e } \\
\text { Faria, Trigueiros e } \\
\text { Ferreira (2012). }\end{array}$ \\
\hline
\end{tabular}

Fonte - Elaborado pelos autores

Como instrumento de pesquisa foi utilizado o questionário, com questões fechadas, que foi dividido em três blocos: perfil institucional, contendo caracterização do respondente (questão 1 a 7) e da organização (questão 8 a 12); fatores contingenciais (questão 13 a 31); e artefatos de custos (questão 32 a 44). Quanto aos fatores contingenciais, o constructo foi adaptado dos estudos internacionais evidenciados na Tabela 2 e do estudo de Espejo (2008), que inclusive consta validado no Brasil.

Para aplicação do instrumento de pesquisa, optou-se por encaminhar via e-mail, com contato telefônico prévio, os questionários aos membros que ocupavam os cargos de administrador, contador, controller, diretor financeiro ou outros, desde que fossem gerentes. Os dados foram coletados entre 2 de novembro e 31 de dezembro de 2015.

Os dados coletados foram tabulados em planilhas eletrônicas e analisados com auxílio de técnicas de análise estatística. Primeiramente foi realizada a análise descritiva. Na sequência, foi utilizada a correlação canônica. Para análise descritiva empregou-se o software Statistical Package for Social Science (SPSS), versão 22.0. Já a correlação canônica foi feita com utilização do software STATGRAPHICS Centurion, para verificar a correlação entre as variáveis pesquisadas.

\section{ANÁLISE DOS DADOS}

\section{Análise descritiva}

Observou-se nas características dos respondentes que, do total de 40 gestores que participaram da pesquisa, 62,5\% (25) são do gênero masculino, a maioria está na faixa de idade entre 26 e 35 anos (35\%) e acima de 45 anos (32,5\%). Foi constatado que $45 \%$ (18) ocupam a função de gerente e $40 \%$ (16), a função de contador/controller, sendo que a maioria dos pesquisados atuam na empresa há um período superior a dez anos (35\%). Porém, quanto ao tempo de ocupação do cargo atual, o destaque fica para os que estão no cargo há até três anos, com $40 \%$ (16). Os resultados revelam que, apesar de um tempo longo de atuação na empresa, a maioria ocupa o cargo atual de gestor há pouco tempo. 
Em relação às características dos hotéis pesquisados, observou-se que a maioria tem de 11 a 20 (30\%) ou de 21 a 40 (30\%) colaboradores. Apenas 15\% (6) dos hotéis contam com mais de 100 funcionários. Foi constatado também que as taxas predominantes de ocupação anual dos leitos são de $31 \%$ a 50\% para 32,5\% (13) dos hotéis, e de acima de 70\% para 37,5\% (15). Observa-se que a maioria das organizações ocupa mais da metade da capacidade de prestação de serviço instalada nos hotéis.

Os resultados revelaram ainda que a maioria dos hotéis tem entre 11 e 20 anos (25\%) de existência ou mais de 30 anos (40\%), sendo que 52,5\% (21) não possuem um setor de contabilidade interna. Após a caracterização inicial dos respondentes da pesquisa, bem como estabelecido o perfil dos hotéis que compõem a amostra analisada neste estudo, procedeu-se à estatística descritiva relacionada aos fatores contingenciais. A Tabela 1 apresenta os resultados da análise da variável porte.

Tabela 1 - Análise descritiva da variável porte organizacional

\begin{tabular}{|l|c|c|c|}
\hline \multicolumn{1}{|c|}{ Variáveis porte organizacional } & $\boldsymbol{F i}$ & $\boldsymbol{f i}$ & $\boldsymbol{f i}$ acumulado \\
\hline Até 50 unidades habitacionais - pequeno porte & 6 & $15,00 \%$ & $15,00 \%$ \\
\hline De 51 a 100 unidades habitacionais- médio porte & 11 & $27,50 \%$ & $42,50 \%$ \\
\hline Acima de 100 unidades habitacionais - grande porte & 23 & $57,50 \%$ & $100,00 \%$ \\
\hline
\end{tabular}

Fonte - Dados da pesquisa

Com relação ao porte da empresa (Tabela 1), constata-se que a maioria dos hotéis $(57,5 \%)$ possui mais de 100 unidades habitacionais, sendo considerados como de grande porte no grupo de organizações analisadas. Na Tabela 2 evidenciam-se os resultados encontrados para as variáveis de ambiente, tecnologia, estrutura e estratégia.

Tabela 2 - Análise descritiva das variáveis ambiente, tecnologia, estrutura e estratégia

\begin{tabular}{|c|c|c|c|c|c|c|}
\hline \multicolumn{2}{|r|}{ Variáveis } & Média & Med & Mín & Máx & $\begin{array}{l}\text { Desvio } \\
\text { Padrão }\end{array}$ \\
\hline \multirow{5}{*}{ 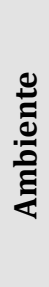 } & Atitudes da concorrência & 3,68 & 4 & 1 & 5 & 1,095 \\
\hline & Competição por mão-de-obra & 3,38 & 4 & 1 & 5 & 1,213 \\
\hline & Tecnologia aplicada à prestação de serviço & 3,13 & 3 & 1 & 5 & 1,305 \\
\hline & Restrições legais, políticas e econômicas do setor & 3,55 & 4 & 1 & 5 & 1,037 \\
\hline & Gostos e preferências dos clientes do setor & 3,43 & 4 & 1 & 5 & 1,217 \\
\hline
\end{tabular}

Legenda: 1 = muito estável, 2 = estável, 3 = intermediário, 4 = dinâmico, 5 = muito dinâmico.

\begin{tabular}{|c|c|c|c|c|c|c|}
\hline \multirow{4}{*}{ 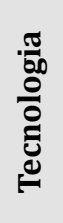 } & Comércio eletrônico & 3,90 & 5 & 1 & 5 & 1,464 \\
\hline & CRM (gerenciamento da relação com o cliente) & 3,43 & 4 & 1 & 5 & 1,631 \\
\hline & Armazenamento de dados & 4,10 & 5 & 2 & 5 & 1,057 \\
\hline & Troca de dados de forma eletrônica & 3,98 & 4 & 1 & 5 & 1,209 \\
\hline
\end{tabular}

Legenda: 1 = não há uso, 2 = está estudando a viabilidade de uso, 3 = uso em baixa intensidade, 4 = uso em moderada intensidade, 5 = uso em grande intensidade. 
Tabela 2 - Continuação

\begin{tabular}{|l|c|c|c|c|c|}
\hline \multicolumn{1}{|c|}{ Variáveis } & Média & Med & Mín & Máx & $\begin{array}{c}\text { Desvio } \\
\text { Padrão }\end{array}$ \\
\hline Ampliação ou oferta de novos serviços & 2,98 & 3,5 & 1 & 5 & 1,368 \\
\hline Contratação e demissão de pessoal & 3,70 & 4 & 1 & 5 & 1,324 \\
\hline \multirow{2}{\text{SE}}{ Seleção de investimentos relevantes } & 2,80 & 3 & 1 & 5 & 1,344 \\
\hline Alocação orçamentária & 2,85 & 3 & 1 & 5 & 1,406 \\
\hline Decisões de preços & 2,65 & 3 & 1 & 5 & 1,424 \\
\hline
\end{tabular}

Legenda: 1 = nunca delega, 2 = já delegou, mas não delega mais, 3 = delega pouco, 4 = delega frequentemente, 5 = delegação total.

\begin{tabular}{|c|c|c|c|c|c|c|}
\hline \multirow{4}{*}{ } & $\begin{array}{l}\text { Buscar alta qualidade do serviço maior que a } \\
\text { concorrência }\end{array}$ & 4,03 & 5 & 2 & 5 & 1,143 \\
\hline & $\begin{array}{l}\text { Desenvolver características diferenciadas do } \\
\text { serviço }\end{array}$ & 3,63 & 4 & 1 & 5 & 1,353 \\
\hline & $\begin{array}{l}\text { A imagem do hotel ser melhor do que o da } \\
\text { concorrência }\end{array}$ & 4,03 & 5 & 2 & 5 & 1,166 \\
\hline & $\begin{array}{l}\text { Ter um preço de serviço menor do que o da } \\
\text { concorrência }\end{array}$ & 2,93 & 3 & 1 & 5 & 1,639 \\
\hline
\end{tabular}

Legenda: 1 = discordo totalmente, 2 = discordo, 3 = nem discordo, nem concordo, 4 = concordo, 5 = concordo totalmente.

Legenda: Med = Mediana; Mín = Mínimo; Máx = Máximo.

Fonte - Dados da pesquisa

De acordo com a média evidenciada na Tabela 2, os resultados das variáveis de ambiente estão equilibrados, porém, nas "atitudes da concorrência" e nas "restrições legais, políticas e econômicas do setor", o dinamismo está mais presente do que a estabilidade. Estes resultados revelam que hotéis participantes da pesquisa percebem de maneira diferente o ambiente em que estão inseridos, razão pela qual espera-se que haja uma distinção entre as configurações do sistema de controle gerencial utilizados por tais organizações, conforme preconizado por Otley (1980) e Chenhall (2007).

Com base na média e no baixo desvio padrão das variáveis de tecnologia, percebe-se que o "armazenamento de dados", a "troca de dados de forma eletrônica" e o "comércio eletrônico" (página na internet que viabiliza comércio eletrônico dos seus serviços) predominam na escala de uso em moderada intensidade desses mecanismos na atividade hoteleira. A este respeito, Espejo (2008) já destacava que há uma tendência crescente de utilização do $e$-commerce como instrumento tecnológico, como um meio de destaque para o canal de comunicação com o mercado.

Nas variáveis do constructo estrutura, tendo como foco a especificação da delegação de autoridade, os resultados apresentados revelam por meio da média e do baixo desvio padrão que o nível de delegação de autoridade é maior ao "contratar e demitir funcionários", em que a delegação ocorre de forma frequente. Nas demais variáveis, percebe-se que os hotéis delegam pouco. Estes resultados também corroboram a identificação de diferenças observadas entre os hotéis pesquisados, o que pode resultar na adoção de diferentes estruturas de controle gerencial nessas organizações. 
$\mathrm{Na}$ análise das variáveis do constructo estratégia, o que mais os hotéis parecem enfatizar em suas estratégias são a busca pela alta qualidade dos serviços, uma imagem do nome do hotel superior à da concorrência e o desenvolvimento de características diferenciadas do serviço. Esses resultados corroboram o preconizado por Chenhall e Langfield-Smith (1998), Hansen e Van der Stede (2004) e Espejo (2008). Nesse contexto, pode-se concluir que os hotéis pesquisados apresentam ênfase na estratégia direcionada para a diferenciação e qualidade, em vez de preços menores do que os da concorrência.

A Tabela 3 evidencia o grau de adoção dos artefatos tradicionais e modernos de custos.

Tabela 3 - Grau de adoção dos artefatos tradicionais e modernos de custos

\begin{tabular}{|c|c|c|c|c|c|c|c|c|c|c|c|c|c|c|}
\hline \multirow[t]{2}{*}{ Artefatos } & \multicolumn{2}{|c|}{$\begin{array}{l}\text { Não há } \\
\text { planos }\end{array}$} & \multicolumn{2}{|c|}{$\begin{array}{l}\text { Avaliou e } \\
\text { descartou }\end{array}$} & \multicolumn{2}{|c|}{$\begin{array}{c}\text { Em } \\
\text { processo } \\
\text { de } \\
\text { avaliação }\end{array}$} & \multicolumn{2}{|c|}{$\begin{array}{c}\text { Adoção } \\
\text { inicial }\end{array}$} & \multicolumn{2}{|c|}{$\begin{array}{c}\text { Adoção } \\
\text { plena }\end{array}$} & \multicolumn{2}{|c|}{ Total } & \multirow[t]{2}{*}{ Méd } & \multirow[t]{2}{*}{ DP } \\
\hline & $F i$ & fi & $F i$ & $f i$ & $F i$ & $f i$ & $F i$ & $f i$ & $F i$ & $f i$ & $F i$ & $f i$ & & \\
\hline \multicolumn{15}{|c|}{ Tradicionais } \\
\hline $\mathbf{C P}$ & 7 & $17,50 \%$ & 2 & $5,00 \%$ & 9 & $22,50 \%$ & 7 & $17,50 \%$ & 15 & $37,50 \%$ & 40 & $100 \%$ & 3,53 & 1,485 \\
\hline CA & 4 & $10,00 \%$ & 2 & $5,00 \%$ & 6 & $15,00 \%$ & 5 & $12,50 \%$ & 23 & $57,5 \%$ & 40 & $100 \%$ & 4,03 & 1,368 \\
\hline $\mathrm{CV}$ & 5 & $12,50 \%$ & 6 & $15,00 \%$ & 5 & $12,50 \%$ & 12 & $30,00 \%$ & 12 & $30,00 \%$ & 40 & $100 \%$ & 3,50 & 1,396 \\
\hline \multicolumn{15}{|c|}{ Modernos } \\
\hline $\mathrm{ABC}$ & 15 & $37,50 \%$ & 1 & $2,50 \%$ & 2 & $5,00 \%$ & 8 & $20,00 \%$ & 14 & $35,00 \%$ & 40 & $100 \%$ & 3,13 & 1,786 \\
\hline TDABC & 21 & $52,50 \%$ & 7 & $17,50 \%$ & 9 & $22,50 \%$ & 2 & $5,00 \%$ & 1 & $2,50 \%$ & 40 & $100 \%$ & 1,88 & 1,091 \\
\hline CM & 15 & $37,50 \%$ & 1 & $2,50 \%$ & 6 & $15,00 \%$ & 7 & $17,50 \%$ & 11 & $27,50 \%$ & 40 & $100 \%$ & 2,95 & 1,694 \\
\hline
\end{tabular}

Legenda: $\mathrm{CP}=$ Custeio Padrão; $\mathrm{CA}=$ Custeio por Absorção; $\mathrm{CV}=$ Custeio Variável; $\mathrm{ABC}=$ Custeio Baseado em Atividades; TDABC = Custeio Baseado em Atividade e Tempo; $\mathrm{CM}=$ Custeio Meta; Méd = Média; DP = Desvio Padrão.

Fonte - Dados da pesquisa

De acordo com a Tabela 3, a adoção plena de artefatos tradicionais nos hotéis tem predominância no Custeio por Absorção com 57,5\% (23), no Custeio Padrão com 37,5\% (15) e no Custeio Variável com 30\% (12). Com base na média, o Custeio por Absorção apresenta o maior resultado, com ênfase para a adoção inicial. Na análise dos artefatos modernos, há predominância da não existência de planos para adoção, sendo 52,5\% (21) para o TDABC e 37,5\% (15) para o ABC e Custeio Meta. A maior média é a do $\mathrm{ABC}$, com tendência para o processo de avaliação para fins de adoção.

De acordo com os resultados acima, o ABC com a maior média nos artefatos modernos para fins de adoção revela tendência para uma futura uniformização 
na adoção. Um sistema uniforme de contabilidade fornece orientação e informação padronizada não apenas para os profissionais ligados a contabilidade, mas também para os gestores, proprietários, empresários e demais usuários da informação contábil (Raymond \& Defranco, 2015). Considerado menos arbitrário, a literatura mostra que o $\mathrm{ABC}$ tem se apresentado relevante nas situações em que é necessário analisar processos que envolvem reestruturações e aperfeiçoamentos dos serviços prestados (Abbas, Golçalves, \& Leoncine, 2012; Guerreiro et al., 2011).

Com base nestes resultados, observa-se uma elevada taxa de adoção aos artefatos tradicionais e uma reduzida taxa quanto aos artefatos modernos, o que corrobora os achados de Lima, Egito e Silva (2004), de Leitão e Silva (2009) e de Faria et al. (2012), sendo que este último estudo foi realizado na rede hoteleira da região do Algarve, em Portugal. Porém, no estudo de Faria et al. (2012), o artefato mais adotado identificado foi o Custeio Variável.

Os resultados anteriores permitem afirmar que o ambiente dos hotéis que participaram da pesquisa é dinâmico ou muito dinâmico, há um uso moderado de tecnologias, delega-se autoridade com frequência para contratação e demissão de pessoal, a maioria dos hotéis é de grande porte e estão focados na alta qualidade, na diferenciação dos serviços e na imagem do hotel. Constatouse também que há uma preferência por artefatos de custos tradicionais. Porém, quanto aos artefatos modernos $\mathrm{ABC}$ e Custeio Meta, há uma tendência futura de maior adoção.

\section{Correlação canônica}

A influência de fatores contingenciais nos artefatos tradicionais e modernos de custos na rede hoteleira do estado do Paraná foi verificada com a correlação canônica, sendo de forma preliminar aplicado o Alfa de Cronbach para verificar a consistência das variáveis do constructo. Segundo Hair, Black, Babin, Anderson e Tatham (2009), o teste Alfa de Cronbach é um tipo de confiabilidade que é utilizado para avaliar uma escala somada em que várias afirmações são somadas para formar um escore total para um constructo. Hair et al. (2009, p. 100) destacam que o Alfa de Cronbach é uma "medida de confiabilidade que varia de 0 a 1 , sendo os valores de 0,60 a 0,70 considerados o limite inferior de aceitabilidade".

Para o constructo dos fatores contingenciais, os resultados mostraram Alfa de Cronbach de 0,748 para o fator ambiente, 0,924 para o fator tecnologia, 0,975 para o fator estrutura, 0,786 para o fator porte e 0,934 para o fator estratégia. Para o constructo dos artefatos de custos, os resultados apontaram Alfa de Cronbach de 0,644 para os artefatos tradicionais de custos e 0,858 para os artefatos modernos de custos. De acordo com o apresentado, os Alfas de Cronbach dos grupos de variáveis estão dentro do limite de aceitabilidade, sendo que a maioria revela um nível consistência muito bom, com indicador superior a 0,900.

Na sequência, apresenta-se a descrição e a análise dos dados, por meio da correlação canônica, entre os grupos de fatores contingenciais e os artefatos tradicionais e modernos de custos pesquisados. Na Tabela 4 observa-se a correlação canônica do grupo do fator contingencial ambiente com os grupos de variáveis de artefatos de custos estudados. 
Tabela 4 - Correlação canônica do grupo de fator contingencial ambiente com os grupos de artefatos tradicionais e modernos de custos

\begin{tabular}{|c|c|c|c|c|c|c|c|}
\hline Artefatos & $\begin{array}{c}\text { Combinações } \\
\text { lineares }\end{array}$ & $\begin{array}{c}\text { Auto } \\
\text { valor }\end{array}$ & $\begin{array}{c}\text { Correlação } \\
\text { canônica }\end{array}$ & $\begin{array}{c}\text { Lambda } \\
\text { de Wilks }\end{array}$ & $\begin{array}{c}\text { Chi- } \\
\text { quadrado }\end{array}$ & D.F. & P-value \\
\hline & 1 & 0,5993 & $\mathbf{0 , 7 7 4 1}$ & 0,3335 & 37,8758 & 15 & $\mathbf{0 , 0 0 0 9}$ \\
\hline \multirow{2}{*}{ Tradicionais } & 2 & 0,1510 & 0,3886 & 0,8326 & 6,3172 & 8 & 0,6117 \\
\hline & 3 & 0,0191 & 0,1383 & 0,9808 & 0,6663 & 3 & 0,8811 \\
\hline \multirow{2}{*}{ Modernos } & 1 & 0,5845 & 0,7645 & 0,3690 & 34,3924 & 15 & $\mathbf{0 , 0 0 3 0}$ \\
\hline & 2 & 0,0896 & 0,2993 & 0,8882 & 4,0881 & 8 & 0,8491 \\
\hline & 3 & 0,0243 & 0,1559 & 0,9756 & 0,8495 & 3 & 0,8376 \\
\hline
\end{tabular}

Fonte - Dados da pesquisa

Verifica-se que foram calculadas três combinações lineares diferentes (coluna Combinações lineares) para os artefatos tradicionais e três para os modernos, as quais representam o número de variáveis que compõem o grupo artefatos. 0 p-value da primeira combinação linear das variáveis dos artefatos tradicionais e dos modernos apresenta-se significativo ao nível de 5\%, de 0,0009 e 0,0030. 0 maior coeficiente de correlação canônica é dos artefatos tradicionais: 0,7741 desta combinação linear. Com base nesse resultado, considera-se que o fator contingencial ambiente influencia mais na adoção de artefatos tradicionais de custos do que artefatos modernos na rede hoteleira do estado do Paraná e, assim, rejeita-se a hipótese $\mathrm{H}_{1}$.

A Tabela 5 apresenta a correlação canônica do grupo de fator contingencial tecnologia com o grupo artefatos tradicionais e modernos de custos.

Tabela 5 - Correlação canônica do grupo de fator contingencial tecnologia com os grupos de artefatos tradicionais e modernos de custos

\begin{tabular}{|c|c|c|c|c|c|c|c|}
\hline Artefatos & $\begin{array}{c}\text { Combinações } \\
\text { lineares }\end{array}$ & $\begin{array}{c}\text { Auto } \\
\text { valor }\end{array}$ & $\begin{array}{c}\text { Correlação } \\
\text { canônica }\end{array}$ & $\begin{array}{c}\text { Lambda } \\
\text { de Wilks }\end{array}$ & $\begin{array}{c}\text { Chi- } \\
\text { quadrado }\end{array}$ & D.F. & P-value \\
\hline & 1 & 0,7849 & $\mathbf{0 , 8 8 5 9}$ & 0,1891 & 58,2846 & 12 & $\mathbf{0 , 0 0 0 0}$ \\
\hline \multirow{2}{*}{ Tradicionais } & 2 & 0,0735 & 0,2711 & 0,8796 & 4,4896 & 6 & 0,6107 \\
\hline & 3 & 0,0506 & 0,2249 & 0,9493 & 1,8176 & 2 & 0,4030 \\
\hline \multirow{2}{*}{ Modernos } & 1 & 0,7430 & 0,8619 & 0,2387 & 50,1331 & 12 & $\mathbf{0 , 0 0 0 0}$ \\
\hline & 2 & 0,0583 & 0,2416 & 0,9289 & 2,5794 & 6 & 0,8595 \\
\hline & 3 & 0,0134 & 0,1160 & 0,9865 & 0,4743 & 2 & 0,7888 \\
\hline
\end{tabular}

Fonte - Dados da pesquisa

Constata-se na Tabela 5 que a maior correlação existente está na primeira combinação linear, entre o conjunto de variáveis do fator contingencial tecnologia e artefatos tradicionais, com 88,59\%. Destaca-se que o nível descritivo ( $p$-value) da primeira combinação linear foi de 0,0000 , menor que $5 \%$. Com base nestes resultados, o fator contingencial tecnologia influencia mais na adoção de artefatos tradicionais de custos do que artefatos modernos na rede hoteleira do estado do Paraná. Dessa forma, rejeita-se a hipótese $\mathrm{H}_{2}$.

A Tabela 6 apresenta a correlação canônica do grupo de fator contingencial estrutura com o grupo de artefatos de custos estudado. 
Tabela 6 - Correlação canônica do grupo de fator contingencial estrutura com os grupos de artefatos tradicionais e modernos de custos

\begin{tabular}{|c|c|c|c|c|c|c|c|}
\hline Artefatos & $\begin{array}{c}\text { Combinações } \\
\text { lineares }\end{array}$ & $\begin{array}{c}\text { Auto } \\
\text { valor }\end{array}$ & $\begin{array}{c}\text { Correlação } \\
\text { canônica }\end{array}$ & $\begin{array}{c}\text { Lambda } \\
\text { de Wilks }\end{array}$ & $\begin{array}{c}\text { Chi- } \\
\text { quadrado }\end{array}$ & D.F. & P-value \\
\hline \multirow{2}{*}{ Tradicionais } & 1 & 0,7526 & 0,8675 & 0,1592 & 63,3846 & 15 & $\mathbf{0 , 0 0 0 0}$ \\
\hline & 2 & 0,2747 & 0,5241 & 0,6437 & 15,1949 & 8 & 0,0555 \\
\hline \multirow{2}{*}{ Modernos } & 3 & 0,1123 & 0,3352 & 0,8876 & 4,1134 & 3 & 0,2495 \\
\hline & 1 & 0,8384 & $\mathbf{0 , 9 1 5 6}$ & 0,0726 & 90,4455 & 15 & $\mathbf{0 , 0 0 0 0}$ \\
\hline & 2 & 0,4396 & 0,6630 & 0,4500 & 27,5453 & 8 & $\mathbf{0 , 0 0 0 6}$ \\
\hline
\end{tabular}

Fonte - Dados da pesquisa

Observa-se na Tabela 6 que a maior correlação com nível de significância de $5 \%$, está na primeira combinação linear, entre o conjunto de variáveis do fator contingencial estrutura e artefatos modernos, com 91,56\%, o que, de acordo com Hair et al. (2009), indica uma associação positiva muito forte. Com base nesses achados, não é possível rejeitar a hipótese $\mathrm{H3}$, de que o fator contingencial estrutura influencia mais na adoção de artefatos modernos de custos do que artefatos tradicionais na rede hoteleira do estado do Paraná.

A não rejeição da H3 corrobora as evidências encontradas em outros estudos desenvolvidos sob a perspectiva contingencial na área contábil, desenvolvidos em outros contextos organizacionais (Baines \& Langfield-Smith, 2003; Beuren \& Fiorentin, 2014; Chenhall, 2007; Cooper \& Kaplan, 1998; Guerra, 2007; Rodniski \& Souza, 2014; Silva et al. 2014). Estes estudos consideram que a necessidade de alcançar vantagens competitivas, o aumento dos serviços ofertados e a ampliação da estrutura organizacional com novos investimentos impactam na adoção de sistemas de custos, visando métodos diferenciados.

Tabela 7 - Coeficientes para as variáveis canônicas do grupo fator contingencial estrutura e artefatos modernos de custos

\begin{tabular}{|l|l|c|}
\hline \multicolumn{3}{c}{ Fator estrutura e artefatos modernos } \\
\hline \multirow{2}{*}{ Grupos } & Variáveis & Combinação linear \\
\hline & & $\mathbf{1}$ \\
\hline Fator estrutura & AOS & 0,5479 \\
\hline & CDP & 0,0578 \\
\hline & SIR & 0,6826 \\
\hline Artefatos modernos & ALO & 0,2357 \\
\hline & DDP & $-0,5063$ \\
\hline & ABC & 1,0207 \\
\hline & TDABC & 0,0270 \\
\hline & CM & $-0,0419$ \\
\hline
\end{tabular}

Observa-se na Tabela 7 que os coeficientes canônicos do grupo fator estrutura e artefatos modernos apresentam uma tendência a maior delegação de autoridade para os gerentes das áreas de decisão da organização na seleção de 
investimentos relevantes (SIR) $(0,6826)$ e na ampliação ou oferta de novos serviços (AOS) $(0,5479)$ são determinantes da maior adoção do artefato moderno ABC $(1,0207)$ e da menor adoção do artefato moderno Custeio Meta $(-0,0419)$. De acordo com Sulaiman, Nazli Nik Ahmad e Alwi (2004), o ABC tende a ser o melhor artefato para contribuir no aumento da capacidade das entidades em cumprirem seus objetivos.

A Tabela 8 evidencia a correlação canônica do grupo de fator contingencial porte com os grupos de artefatos estudados.

Tabela 8- Correlação canônica do grupo de fator contingencial porte com os grupos de artefatos tradicionais e modernos de custos

\begin{tabular}{|c|c|c|c|c|c|c|c|}
\hline Artefatos & $\begin{array}{c}\text { Combinação } \\
\text { linear }\end{array}$ & $\begin{array}{c}\text { Auto } \\
\text { valor }\end{array}$ & $\begin{array}{c}\text { Correlação } \\
\text { canônica }\end{array}$ & $\begin{array}{c}\text { Lambda } \\
\text { de Wilks }\end{array}$ & $\begin{array}{c}\text { Chi- } \\
\text { quadrado }\end{array}$ & D.F. & P-value \\
\hline Tradicionais & 1 & 0,6590 & 0,8118 & 0,3409 & 39,2769 & 3 & $\mathbf{0 , 0 0 0 0}$ \\
\hline Modernos & 1 & 0,4815 & 0,6939 & 0,5184 & 23,9785 & 3 & $\mathbf{0 , 0 0 0 0}$ \\
\hline \multicolumn{7}{c}{ Fonte - Dados da pesquisa } \\
\end{tabular}

A Tabela 8 evidencia que a maior correlação com nível de significância de 5\% está na combinação linear entre o conjunto de variáveis do fator contingencial porte e artefatos tradicionais, com $81,18 \%$. Este achado indica a rejeição da hipótese $\mathrm{H}_{4}$, de que o fator contingencial porte organizacional influencia mais na adoção de artefatos modernos de custos do que artefatos tradicionais na rede hoteleira do estado do Paraná.

A Tabela 9 apresenta a correlação canônica do grupo de fator contingencial estratégia com o grupo de artefatos tradicionais e modernos de custos.

Tabela 9 - Correlação canônica do grupo de fator contingencial estratégia com os grupos de artefatos tradicionais e modernos de custos

\begin{tabular}{|l|c|c|c|c|c|c|c|}
\hline Artefatos & $\begin{array}{c}\text { Combinações } \\
\text { lineares }\end{array}$ & $\begin{array}{c}\text { Auto } \\
\text { valor }\end{array}$ & $\begin{array}{c}\text { Correlação } \\
\text { canônica }\end{array}$ & $\begin{array}{c}\text { Lambda } \\
\text { de Wilks }\end{array}$ & $\begin{array}{c}\text { Chi- } \\
\text { quadrado }\end{array}$ & D.F. & P-value \\
\hline \multirow{2}{*}{ Tradicionais } & 1 & 0,7047 & 0,8394 & 0,2693 & 45,9065 & 12 & $\mathbf{0 , 0 0 0 0}$ \\
\hline & 2 & 0,0635 & 0,2521 & 0,9122 & 3,2133 & 6 & 0,7816 \\
\hline \multirow{2}{*}{ Modernos } & 3 & 0,0257 & 0,1605 & 0,9742 & 0,9134 & 2 & 0,6334 \\
\hline & 1 & 0,7383 & 0,8592 & 0,2270 & 51,8834 & 12 & $\mathbf{0 , 0 0 0 0}$ \\
\hline & 2 & 0,1242 & 0,3524 & 0,8678 & 4,9611 & 6 & 0,5488 \\
\hline
\end{tabular}

Fonte - Dados da pesquisa

Observa-se que a maior correlação com nível de significância de 5\% está na primeira combinação linear, entre o conjunto de variáveis do fator contingencial estratégia e artefatos modernos, com 85,92\%, o que, de acordo com Hair et al. (2009), indica uma associação positiva alta. Estes achados não permitem rejeitar a hipótese $\mathrm{H}_{5}$, de que o fator contingencial estratégia influencia mais na adoção de artefatos modernos de custos do que artefatos tradicionais na rede hoteleira do estado do Paraná. 
Os resultados corroboram as evidências encontradas em outros contextos (Baines \& Langfield-Smith, 2003; Beuren \& Fiorentin, 2014; Chenhall, 2007; Cooper \& Kaplan, 1998; Guerra, 2007; Rodniski \& Souza, 2014; Silva et al., 2014). As evidências destes estudos mostram que a busca por sistemas de custos diferenciados, que sejam considerados atuais e úteis, tendem a suprir a necessidade informacional dos gestores, contribuindo para o desenvolvimento de características diferenciadas dos serviços, o aumento da qualidade dos serviços e a imagem organizacional.

Tabela 10 - Coeficientes para as variáveis canônicas do grupo fator contingencial estratégia e artefatos modernos de custos

\begin{tabular}{|c|c|c|}
\hline \multicolumn{3}{|c|}{ Fator estratégia e artefatos modernos } \\
\hline \multirow{2}{*}{ Grupos } & Variáveis & Combinação linear \\
\hline \multirow{3}{*}{ Fator estratégia } & BAQ & $\mathbf{1}$ \\
\hline & DCPU & 0,2544 \\
\hline \multirow{3}{*}{ Artefatos modernos } & IMMC & 0,3906 \\
\hline & PSMC & 0,2424 \\
\hline & ABC & $-0,2016$ \\
\hline & TDABC & 0,9718 \\
\hline & Fonte - Dados da pesquisa & $-0,0155$ \\
\hline
\end{tabular}

Observa-se na Tabela 10 que os coeficientes correspondentes ao primeiro par canônico do grupo fator estratégia e artefatos modernos apresentam uma tendência a maior importância em desenvolver características diferenciadas do serviço (DCPU) $(0,3906)$ e em buscar alta qualidade do serviço, muito maior do que o da concorrência (BAQ) $(0,2544)$. Eles são determinantes da maior adoção do artefato moderno $\operatorname{ABC}(0,9718)$ e da menor adoção do $\operatorname{TDABC}(-0,0155)$.

Em síntese, considerando-se a amostra investigada, observa-se a partir dos resultados encontrados que as variáveis contingenciais ambiente, tecnologia, estrutura, porte organizacional e estratégia podem auxiliar na compreensão de fatores que influenciam na adoção de artefatos tradicionais e modernos de custos na gestão de empresas hoteleiras do estado do Paraná, evidências que estimulam a realização de novos estudos sobre esta temática.

\section{CONSIDERAÇÕES FINAIS}

Esta pesquisa objetivou evidenciar a influência de fatores contingenciais na adoção de artefatos tradicionais e modernos de custos na rede hoteleira do estado do Paraná. A abordagem contingencial aplicada para verificar os sistemas de custeio adotados por hotéis dessa região do país possibilita uma nova visão das organizações hoteleiras, de modo a entender os fatores que influenciam na adoção de artefatos de custos.

Os resultam encontrados indicaram que a maioria dos hotéis do estado do Paraná adotam de forma plena os artefatos tradicionais de custos, com destaque 
para o Custeio por Absorção. Já quanto aos artefatos modernos, boa parte dos hotéis investigados relataram não possuir planos para suas adoções. Estes resultados são convergentes aos achados encontrados em estudos anteriores, que sugerem a predominância de adoção de artefatos tradicionais, em detrimento de modernos, mesmo que aqueles possam não ser considerados os mais adequados para a tomada de decisões gerenciais.

Dentre as respostas obtidas, constatou-se que alguns gestores relataram que o Custeio Baseado em Atividades e o Custeio Meta apresentam-se em um estágio inicial de adoção em suas organizações. Estes resultados sugerem que, nesta área, futuramente, é possível que tais artefatos sejam utilizados por um número maior de organizações hoteleiras, o que pode revelar uma mudança nos padrões de controle de custos a serem utilizados por essas organizações. Tais evidências estimulam a realização de novos estudos.

Dentre as diferentes variáveis contingenciais utilizadas para se investigar a correlação entre os fatores contingenciais (ambiente, tecnologia, estrutura, porte organizacional e estratégia) e a adoção dos artefatos tradicionais e modernos de custos investigados, os resultados encontrados a partir da correlação canônica sugerem que os fatores contingenciais estrutura e estratégia são os que influenciam mais na adoção de artefatos modernos de custos do que de tradicionais na rede hoteleira do estado do Paraná, o que sustenta a não rejeição das Hipóteses $\mathrm{H}_{3}$ e $\mathrm{H}_{5}$. Tanto o fator contingencial estrutura quanto o fator estratégia são determinantes da maior adoção do artefato moderno ABC. Desta forma, pode-se concluir que tais variáveis auxiliam na compreensão de fatores que influenciam na adoção de artefatos tradicionais e modernos de custos na gestão de empresas hoteleiras do estado do Paraná.

Apesar das potenciais limitações, esta pesquisa fornece evidências que contribuem para a literatura da área, tendo em vista a não identificação de estudos semelhantes desenvolvidos sob a configuração proposta nesta pesquisa, no contexto observado. Contribui em função da evidenciação da influência de fatores contingenciais sobre a adoção de artefatos tradicionais e modernos de custos em organizações hoteleiras, gerando informações que podem estimular a reflexão sobre oportunidades de melhorias e avanços nas práticas de gestão adotadas por essas organizações. Como o estudo limitou-se à análise de uma amostra estabelecida no estado do Paraná, para a realização de futuras pesquisas sugere-se a inclusão de organizações hoteleiras de outros estados e regiões do país, a fim de se verificar similaridades e diferenças, bem como comparações que oportunizem a compreensão de fatores que influenciam na adoção de artefatos modernos de contabilidade gerencial e a consolidação de um sistema uniforme de contabilidade de hotéis.

\section{REFERÊNCIAS}

Abbas, K., Gonçalves, M. N., \& Leoncine, M. (2012). Os métodos de custeio: vantagens, desvantagens e sua aplicabilidade nos diversos tipos de organizações apresentadas pela literatura. Contexto, 12(22), 145-159.

Abdel-Kader, M., \& Luther, R. (2006). IFAC's conception of the evolution of management accounting. Advances in management accounting, 15(1), 229-247. 
Abdel-Kader, M., \& Luther, R. (2008). The impact of firm characteristics on management accounting practices: A UK-based empirical analysis. The British Accounting Review, 40(1), 2-27.

Ansoff, H. I., \& Mcdonnell, E. J. (1993). Implantando a administração estratégica (2ª ed.). São Paulo: Atlas.

Baines, A., \& Langfield-Smith, K. (2003). Antecedents to management accounting change: a structural equation approach. Accounting, Organizations and Society, 28(7-8), 675-698.

Beuren, I. M., \& Fiorentin, M. (2014). Influência de fatores contingenciais nos atributos do sistema de contabilidade gerencial: um estudo em empresas têxteis do estado do Rio Grande do Sul. Revista de Ciências da Administração, 16(38), 195-212.

Burns, J., \& Vaivio, J. (2001). Management accounting change. Management accounting research, 12(4), 389-402.

Chenhall, R. H. (2003). Management control systems design within its organizational context: findings from contingency-based research and directions for the future. Accounting, Organizations and Society, 28(2-3), 127-168.

Chenhall, R. H. (2007). Theorizing contingencies in management control systems research. Handbooks of Management Accounting Research, 1, 163-205.

Chenhall, R. H., \& Langfield-Smith, K. (1998). Adoption and benefits of management accounting practices: an Australian study. Management Accounting Research, 9(1), 1-19.

Cooper, D., \& Schindler, P. S. (2003). Métodos de pesquisa em administração (7aㅡ ed.). Porto Alegre, RS: Bookman.

Cooper, R., \& Kaplan, R. S. (1998). The promise--and peril--of integrated cost systems. Harvard Business Review, 76(4), 109-119.

Dekker, H. C., Groot, T., \& Schoute, M. (2012). A Balancing Act? The Implications of Mixed Strategies for Performance Measurement System Design. Journal of Management Accounting Research, 25, 71-98.

Espejo, M. M. S. B. (2008). Perfil dos atributos do sistema orçamentário sob a perspectiva contingencial: uma abordagem multivariada. Tese de Doutorado, Faculdade de Economia, Administração e Contabilidade, Universidade de São Paulo, São Paulo.

Espejo, M. M. S. B., Costa, F., Cruz, A. P. C., \& Almeida, L. B. (2009). Uma análise críticoreflexiva da compreensão da adoção dos artefatos de contabilidade gerencial sob uma lente alternativa: a contribuição de abordagens organizacionais. Revista de Contabilidade e Organizações, 3(5), 25-43.

Faria, A. R., Trigueiros, D., \& Ferreira, L. (2012). Práticas de custeio e controlo de gestão no sector hoteleiro do Algarve. Tourism \& Management Studies, 8(1), 100-107.

Frezatti, F. (2005). Management accounting profile of firms located in Brazil: a field study. Brazilian Administration Review, 2(1), 73-87.

Fonseca, J. S., \& Martins, G. A. (1996). Curso de estatística. São Paulo: Atlas.

Gordon, L. A., \& Narayanan, V. K. (1984). Management accounting systems, perceived environmental uncertainty and organization structure: an empirical investigation. Accounting, Organizations and Society, 9(1), 33-47.

Guerra, A. R. (2007). Arranjos entre fatores situacionais e sistema de contabilidade gerencial sob a ótica da teoria da contingência. Dissertação de Mestrado, Faculdade de Economia, Administração e Contabilidade, Universidade de São Paulo, São Paulo.

Guerreiro, R., Cornachione Júnior, E. B., \& Soutes, D. O. (2011). Empresas que se destacam pela qualidade das informações a seus usuários externos também se destacam pela utilização de artefatos modernos de contabilidade gerencial? Revista Contabilidade \& Finanças, 22(55), 88-113. 
Guerreiro, R., Frezatti, F., Lopes, A. B., \& Pereira, C. A. (2005). 0 entendimento da contabilidade gerencial sob a ótica da teoria institucional. Organizações \& Sociedade, 12(35), 91-106.

Hair, J. F., Black, W. C., Babin, B. J., Anderson, R. E., \& Tatham, R. L. (2009). Análise multivariada de dados (6 ${ }^{\mathrm{a}} \mathrm{ed}$.). Porto Alegre, RS: Bookman.

Hansen, S. C., \& Van der Stede, W. A. (2004). Multiple facets of budgeting: an exploratory analysis. Management Accounting Research, 15(4), 415-439.

Hyvönen, J. (2007). Strategy, performance measurement techniques and information technology of the firm and their links to organizational performance. Management Accounting Research, 18(3), 343-366.

Johnson, H. T., \& Kaplan, R. S. (1993). Contabilidade gerencial: a restauração da relevância da contabilidade nas empresas. Rio de Janeiro: Campus.

Leitão, C. R. S., \& Silva, J. D. G. (2009). Utilização do custeio variável no gerenciamento de hotéis: uma pesquisa no setor hoteleiro do nordeste brasileiro. Contabilidade Vista \& Revista, 17(3), 25-43.

Lima, G. A. S. F., Egito, M. O. T., \& Silva, J. D. G. (2004). Utilização de informações de custos no processo gerencial: estudo comparativo entre a hotelaria do estado do Rio Grande do Norte e a região nordeste, sob a ótica da gestão econômico-financeira. Revista Contabilidade \& Finanças, 15(spe), 106-116.

Maher, M. (2001). Contabilidade de custos: criando valor para a administração (J. E. Santos, trad.). São Paulo: Atlas.

Marquesan, F. F. S., Guzzo, R. F., Zawislak, P. A., \& Tello-Gamarra, J. (2015). A importância dos ativos específicos na diferenciação de firmas do setor hoteleiro. Revista Economia \& Gestão, 15(41), 79-111.

Martins, E. (2010). Contabilidade de custos (10 ed.). São Paulo: Atlas.

Maximiano, A. C. A. (2004). Introdução à administração (6⿳亠丷厂 ed.). São Paulo: Atlas.

Otley, D. T. (1980). The contingency theory of management accounting: achievement and prognosis. Accounting, Organizations and Society, 5(4), 413-428.

Píccolo, D. R., \& Gândara, J. M. G. (2012). Distribuição espacial da hotelaria de rede no estado do Paraná (Brasil). Turismo e Sociedade, 5(2), 466-488.

Raymond, R. S., \& Defranco, A. (2015). Uniform system of accounts for the lodging industry, 11th revised edition: the new guidelines for the lodging industry. The Journal of Hospitality Financial Management, 23(1), 79-89.

Rodniski, C. M.; Souza, M. A. (2014). Estrutura do sistema de custos e os atributos da informação: um estudo com empresas brasileiras. Revista Universo Contábil, 10(4), 45-67.

Sharma, D. S. (2002). The differential effect of environmental dimensionality, size, and structure on budget system characteristics in hotels. Management Accounting Research, 13(1), 101-130.

Silva, J. D. G. (2000). Investigação da prática da gestão econômica na atividade hoteleira. Tese de Doutorado, Faculdade de Economia, Administração e Contabilidade, Universidade de São Paulo, São Paulo.

Silva, M. Z., Scarpin, J. E., Rocha, W., \& Di Domenico, D. (2014). Fatores contingenciais que contribuem para a decisão de modificação do sistema de custeio: estudo de caso em uma indústria moageira. Revista de Administração, 49(2), 267-279.

Soutes, D. O. (2006). Uma investigação do uso de artefatos da contabilidade gerencial por empresas brasileiras. Dissertação de Mestrado, Faculdade de Economia, Administração e Contabilidade, Universidade de São Paulo, São Paulo. 
Soutes, D. O., \& Zen, M. J. C. M. (2005). Estágios evolutivos da contabilidade gerencial em empresas brasileiras. Anais do Congresso USP de Controladoria e Contabilidade, Brasil, 29.

Souza, M. A., Lisboa, L. P., \& Rocha, W. (2003). Práticas de contabilidade gerencial adotadas por subsidiárias brasileiras de empresas multinacionais. Revista Contabilidade \& Finanças, 14(32), 40-57.

Sulaiman, M. B., Nazli Nik Ahmad, N., \& Alwi, N. (2004). Management accounting practices in selected asian countries: a review of the literature. Managerial Auditing Journal, 19(4), 493-508.

Tsai, H., Song, H., \& Wong, K. K. (2009). Tourism and hotel competitiveness research. Journal of Travel \& Tourism Marketing, 26(5-6), 522-546.

Wilke, E. P., \& Rodrigues, L. C. (2013). Fontes de pressão institucional: reflexões sobre legitimidade na indústria hoteleira brasileira. Revista Brasileira de Pesquisa em Turismo, 7(2), 337-357.

Zonatto, V. C. S., Schuh, C., \& Zonatto, P. A. F. (2014). Contribuição dos recursos estratégicos nos processos de criação da inovação em uma rede de cooperação hoteleira. Revista Turismo em Análise, 25(3), 700-732.

Recebido em: 29/09/2017

Aprovado em: 19/04/2018

\section{CONTRIBUIÇÕES}

Cleston Alexandre dos Santos: Definição do problema de pesquisa e objetivos, desenvolvimento da proposição teórica, realização da revisão bibliográfica e fundamentação teórica, escolha dos procedimentos metodológicos, coleta de dados, análise de dados, elaboração de tabelas, gráficos e figuras, realização de cálculos e projeções, revisão crítica do manuscrito, redação do manuscrito, adequação do manuscrito às normas da RTA.

Vinícius Costa da Silva Zonatto: Definição do problema de pesquisa e objetivos, desenvolvimento da proposição teórica, realização da revisão bibliográfica e fundamentação teórica, escolha dos procedimentos metodológicos, análise de dados, realização de cálculos e projeções, revisão crítica do manuscrito, redação do manuscrito, adequação do manuscrito às normas da RTA. 